\title{
Studying Interfacial Reactions of Cholesterol Sulfate in an Unsaturated Phosphatidylglycerol Layer with Ozone Using Field Induced Droplet Ionization Mass Spectrometry
}

\author{
Jae Yoon Ko, ${ }^{1}$ Sun Mi Choi, ${ }^{1,2}$ Young Min Rhee, ${ }^{1,2}$ J. L. Beauchamp, ${ }^{3}$ Hugh I. Kim ${ }^{1}$ \\ ${ }^{1}$ Department of Chemistry, Pohang University of Science and Technology (POSTECH), 790-784, Pohang, Republic of Korea \\ ${ }^{2}$ Institute of Theoretical and Computational Chemistry (WCU Institute), Pohang University of Science and Technology \\ (POSTECH), Pohang, Republic of Korea \\ ${ }^{3}$ Noyes Laboratory of Chemical Physics, California Institute of Technology, Pasadena, CA, USA
}

\begin{abstract}
Field-induced droplet ionization (FIDI) is a recently developed ionization technique that can transfer ions from the surface of microliter droplets to the gas phase intact. The air-liquid interfacial reactions of cholesterol sulfate ( $\mathrm{CholSO}_{4}$ ) in a 1-palmitoyl-2-oleoyl-sn-phosphatidylglycerol (POPG) surfactant layer with ozone $\left(\mathrm{O}_{3}\right)$ are investigated using field-induced droplet ionization mass spectrometry (FIDI-MS). Time-resolved studies of interfacial ozonolysis of $\mathrm{CholSO}_{4}$ reveal that water plays an important role in forming oxygenated products. An epoxide derivative is observed as a major product of $\mathrm{CholSO}_{4}$ oxidation in the FIDI-MS spectrum after exposure of the droplet to $\mathrm{O}_{3}$ for $5 \mathrm{~s}$. The abundance of the epoxide product then decreases with continued $\mathrm{O}_{3}$ exposure as the finite number of water molecules at the air-liquid interface becomes exhausted. Competitive oxidation of $\mathrm{CholSO}_{4}$ and POPG is observed when they are present together in a lipid surfactant layer at the air-liquid interface. Competitive reactions of $\mathrm{CholSO}_{4}$ and POPG with $\mathrm{O}_{3}$ suggest that $\mathrm{CholSO}_{4}$ is present with POPG as a well-mixed interfacial layer. Compared with $\mathrm{CholSO}_{4}$ and $\mathrm{POPG}$ alone, the overall ozonolysis rates of both $\mathrm{CholSO}_{4}$ and POPG are reduced in a mixed layer, suggesting the double bonds of both molecules are shielded by additional hydrocarbons from one another. Molecular dynamics simulations of a monolayer comprising POPG and $\mathrm{CholSO}_{4}$ correlate well with experimental observations and provide a detailed picture of the interactions between $\mathrm{CholSO}_{4}$, lipids, and water molecules in the interfacial region.
\end{abstract}

Key words: Field induced droplet ionization, Interfacial chemistry, Ozonolysis, Cholesterol sulfate, Unsaturated phospholipid, Reaction kinetics

Jae Yoon Ko and Sun Mi Choi contributed equally to this work.

Electronic supplementary material The online version of this article (doi:10.1007/s13361-011-0275-9) contains supplementary material, which is available to authorized users.

Correspondence to: Hugh I. Kim; e-mail: hughkim@postech.edu

\section{Introduction}

Cholesterol sulfate $\left(\mathrm{CholSO}_{4}\right)$ is a naturally occurring $\checkmark$ cholesterol (Chol) analogue widely found in various tissues [1-5]. Its physiologic function is only partially understood. However, $\mathrm{CholSO}_{4}$ is generally known to play 
roles in stabilizing and modifying the properties of cell membranes. It protects erythrocytes from osmotic lysis [2] and regulates sperm capacitation [3]. A recent study using mass spectrometry has reported $\mathrm{CholSO}_{4}$ as a potential biomarker of human prostate cancer [4]. The structure of $\mathrm{CholSO}_{4}$ is identical to Chol except for its anionic sulfate functionality. Both molecules exert comparable effects in stabilizing and modifying properties of cell membranes [5]. However, presence or absence of the sulfate moiety also exerts dissimilar effects on cell membranes. For example, studies have shown that $\mathrm{CholSO}_{4}$ increases the order of acyl chains of phosphatidylcholine for temperatures higher than the gel-to-liquid crystal transition point, while it decreases the order for temperatures below the phase transition point [6-8]. A simulation study of interactions of 1,2-dipalmitoyl-sn-phosphatidylcholine (DPPC) with $\mathrm{CholSO}_{4}$ and with Chol reveals that the hydrophobic rings of both species occupy similar locations in acyl chain bilayers [5]. The simulation also demonstrates that the head group of $\mathrm{CholSO}_{4}$ is located slightly below the lipid phosphate group, forming more hydrogen bonds with water molecules than is the case for the hydroxyl group of Chol [5]. This leads to stronger interactions between DPPC and $\mathrm{CholSO}_{4}$, which increases the order effect on DPPC acyl chains, but the effect is limited to adjacent DPPC.

The double bond of the oleoyl group of monounsaturated phospholipid, which is the most common unsaturated lipid found in all membranes, is located at the middle $(\omega-9)$ of the acyl chain [9]. Previous theoretical studies have indicated that this specific location of the cis double-bond allows a maximum surface area per lipid, thus providing the highest fluidity of the lipid layer [9]. The double-bond at $\omega-9$ also weakens condensing and ordering effects induced by Chol $[10,11]$. This effect is proposed to be caused by colocalization of the phospholipid double-bond and the hydrocarbon chain of Chol [9]. In contrast to Chol, interactions of $\mathrm{CholSO}_{4}$ in unsaturated phospholipid layers have not been as thoroughly investigated. As reported in previous studies of a saturated phospholipid membrane, the common hydrophobic rings and tails of $\mathrm{CholSO}_{4}$ and $\mathrm{Chol}$ may exhibit similar interactions with acyl chains of unsaturated phospholipids, with the different head groups being the main source of distinctive behavior.

Field-induced droplet ionization mass spectrometry (FIDI-MS) employs a soft ionization technique to sample ions from the surface of microliter liquid droplets to a mass analyzer [12-15]. Utilizing FIDI-MS, several studies of time-resolved heterogeneous reactions at air-liquid interfaces have been reported $[14,15]$. For example, the location and orientation of SP-B B-25 $_{1}$ (a shortened version of human surfactant protein B) in a lipid surfactant layer has been deduced from experimentally observed reactivity with ozone $\left(\mathrm{O}_{3}\right)$ using a FIDI-MS methodology [14]. In addition, detailed mechanistic studies of the heterogeneous oxidation of unsaturated phospholipids as well as the alteration of phospholipid compositions resulting from reaction with $\mathrm{O}_{3}$ at the air-liquid interface have also been reported [15].

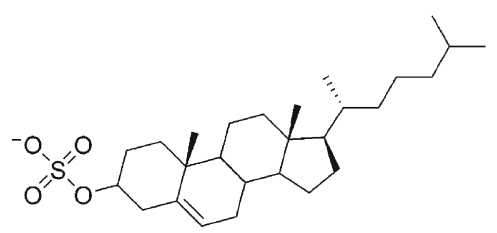

cholesterol sulfate $\left(\mathrm{CholSO}_{4}\right)$

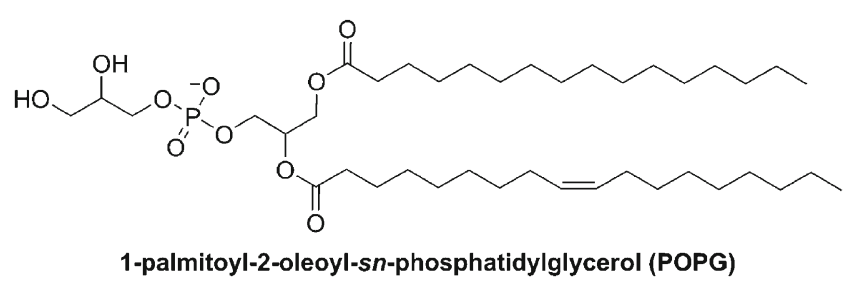

Scheme 1. Structures of $\mathrm{CholSO}_{4}$ and POPG anions investigated in this study

In this study, we investigate the interfacial reactivity of $\mathrm{CholSO}_{4}$ both alone and in the 1-palmitoyl-2-oleoyl-snphosphatidylglycerol (POPG) surfactant layer (Scheme 1) at the air-liquid interface of microliter droplet via heterogeneous oxidation by $\mathrm{O}_{3}$ and computational modeling. $\mathrm{CholSO}_{4}$ is used due to its high ionization efficiency and interesting structural and functional characteristics in a lipid membrane, as well as its high similarity to Chol [16]. Especially, $\mathrm{CholSO}_{4}$ has been reported to be miscible both in liquid condensed and liquid extended layers [16]. The unique capabilities of FIDI-MS provide detailed information regarding reactants, intermediates, and products present in the interfacial layers. First, we probe air-liquid interfacial oxidation of $\mathrm{CholSO}_{4}$ by $\mathrm{O}_{3}$. Then, in order to understand characteristics of the air-liquid interfacial ozonolysis of $\mathrm{CholSO}_{4}$, we have performed solution and solid phase reactions with $\mathrm{O}_{3}$ for comparison purpose. Finally, we investigate the air-liquid interfacial reaction of $\mathrm{CholSO}_{4}$ in the POPG layer with $\mathrm{O}_{3}$. To support our interpretation of the experimental results, molecular dynamics (MD) simulations of $\mathrm{CholSO}_{4}$ mixed with POPG in a monolayer on the surface of water have been performed. The MD simulations correlate well with experimental observations and provide additional insights into the interactions between lipids and water molecules in the interfacial region.

\section{Experimental}

\section{Chemicals and Reagents}

The sodium salt of $\mathrm{CholSO}_{4}$ is purchased from SigmaAldrich (St. Louis, MO, USA), 25-[N-[(7-nitro-2-1,3benzoxadiazol-4-yl)methyl]amino]-27-norcholesterol (25NBD Chol) and the sodium salt of POPG are purchased from Avanti Polar Lipid (Alabaster, AL, USA) and used without further purification. All solvents (water and methanol) are HPLC grade and purchased from EMD Chemicals Inc. (Gibbstown, NJ, USA). 


\section{Air-Liquid Interfacial Oxidation by $\mathrm{O}_{3}$}

The FIDI-MS instrument is based on a design previously described by Grimm and Beauchamp [13]. A stainless steel capillary with $\sim 2 \mathrm{~mm}$ o.d. hanging droplet of analyte solution is located between the atmospheric sampling inlet of a mass analyzer (Thermo Finnigan LCQ Deca mass spectrometer) and a parallel plate electrode. The droplet is exposed to $\mathrm{O}_{3}$ for a desired period of time between 0 to $60 \mathrm{~s}$. FIDI sampling is then achieved by applying pulsed voltages of -4 and $-2 \mathrm{kV}$ to the parallel plate electrode and supporting capillary, respectively. The FIDI-MS spectra reported in this study are obtained by averaging 10 30 individually acquired spectra from separately prepared droplets. Ion abundances are analyzed by measuring peak areas in FIDI-MS spectra. Approximately $20 \mathrm{ppm} \mathrm{O}_{3}$ is generated using a pencil-style UV calibration lamp (model 6035; Oriel). The ozone concentration is measured spectrophotometrically using an absorption cell with $10 \mathrm{~cm}$ path length. The ozone concentration is calculated as $\sim 20 \mathrm{ppm}$ in air with a molar absorption coefficient of $1.15 \times 10^{-17} \mathrm{~cm}^{2}$ molecule ${ }^{-1}$ in a flow that continually bathes the droplet at $\sim 1500 \mathrm{~mL} \mathrm{~min}^{-1} .50 \mu \mathrm{M}$ $\mathrm{CholSO}_{4}$ or a mixture of $50 \mu \mathrm{M} \mathrm{CholSO}_{4}$ and $50 \mu \mathrm{M}$ POPG in 1:1 (by volume) water and methanol feed the droplet source.

\section{Solution Phase and Solid Phase $\mathrm{O}_{3}$ Reactions}

A continuous flow of $\sim 20 \mathrm{ppm} \mathrm{O}_{3}$ in $\mathrm{He}$ is bubbled into a $50 \mu \mathrm{M}$ $\mathrm{CholSO}_{4}$ solution $(200 \mu \mathrm{L})$ in 1:1 (by volume) water and methanol solvent for $15,30,45$, and $60 \mathrm{~s}$ for solution phase $\mathrm{O}_{3}$ reactions. For solid phase reactions, a continuous flow of $\sim 20 \mathrm{ppm} \mathrm{O}_{3}$ in $\mathrm{He}$ is applied to a dried $\sim 1.4 \times 10^{-4} \mathrm{~g}$ of $\mathrm{CholSO}_{4}$ film in $20 \mathrm{~mL}$ glass vial for $30 \mathrm{~min}, 2 \mathrm{~h}$, and $12 \mathrm{~h}$. The dried $\mathrm{CholSO}_{4}$ film is prepared by drying $300 \mu \mathrm{L}$ of $1 \mathrm{mM} \mathrm{CholSO}_{4}$ solution dissolved in 2:1 (by volume) chloroform and ethanol solvent under dry $\mathrm{N}_{2}$. The film is then placed under vacuum overnight. For analysis a sample solution is prepared with a total $50 \mu \mathrm{M}$ concentration using methanol for electrospray ionization (ESI). Product analysis is performed on a Thermo Scientific LTQ Velos dual ion trap mass spectrometer in negative ion mode.

\section{Fluorescence Microscopy}

The fluorescence labeled $\mathrm{CholSO}_{4}\left(25-\mathrm{NBD} \mathrm{CholSO}_{4}\right)$ is prepared from 25-NBD cholesterol using modified method of Duff [17] by Sandhoff et al. [18]. In brief, $0.355 \mathrm{mg}$ of 25-NBD cholesterol is dissolved $20 \mu \mathrm{L}$ of $5 \mathrm{mg} / \mathrm{mL}$ sulfur trioxide pyridine complex solution in absolute pyridine. After $10 \mathrm{~min}$ in room temperature, $2.1 \mu \mathrm{L}$ of $314.1 \mathrm{mM}$ barium sulfate is added and left for $10 \mathrm{~min}$ in room temperature. Then, the sample is incubated in distilled water in the refrigerator $\left(4^{\circ} \mathrm{C}\right)$ for $1 \mathrm{~h}$. Lastly, the solution is centrifuged at 10,000 rpm for $10 \mathrm{~min}$ at $15^{\circ} \mathrm{C}$ and stored at $-20^{\circ} \mathrm{C}$. The purity of the derived 25-NBD $\mathrm{CholSO}_{4}$ is checked using TLC and single spot is found from normal phase chromatography. Fluorescence microscopy ob- servation of air-liquid interface is carried out using fluorescence microscope (Eclipse 80i; Nikon) with a mercury lamp as a light source. Lipid monolayer is prepared on the $300 \mu \mathrm{L}$ water or water/methanol (1:1 by volume) droplet, which is deposited on the microscope slide with a cavity. The lipid layer composed with the mixture of $10 \mu \mathrm{M} \mathrm{CholSO}_{4}$ and $10 \mu \mathrm{M}$ POPG contains $0.5 \mathrm{~mol} \%$ of $25-\mathrm{NBD}$ cholesterol sulfate.

\section{Computational Modeling}

Molecular dynamics (MD) simulations are performed with the all-atom CHARMM22 force field [19, 20] using the CHARMM package [21]. Flexible TIP3P water potential is used with Hooke's constants of $900 \mathrm{kcal} \mathrm{mol}^{-1} \AA^{-2}$ for the $\mathrm{OH}$ bond and $110 \mathrm{kcal} \mathrm{mol}^{-1} \mathrm{rad}^{-2}$ for the H-O-H angle. Models of $\mathrm{CholSO}_{4}$ and POPG lipid monolayer-water system consist of 48 hexagonally-packed lipids and are simulated at different surface densities of $55,60,65$, and 70 $\AA^{2}$ per lipid molecule. A wall potential described by the repulsive part of $V=\varepsilon\left[2 / 15(\sigma / \mathrm{r})^{9}-(\sigma / \mathrm{r})^{3}\right]$ with $\varepsilon=0.1521 \mathrm{kcal} /$ mol and $\sigma=3.1538 \AA$ is employed to prevent water molecules from diffusing out of the box. The force field parameters of $\mathrm{CholSO}_{4}$ are generated based on allatom Chol parameter set reported by Pitman et al [22]. The modifications are on the head group of $\mathrm{CholSO}_{4}$ : the parameters of $\mathrm{S}$ and $\mathrm{O}$ atoms are directly adopted from methylsulfate parameters [23, 24] and the charge on the linking carbon on the cholesteryl ring is adjusted to the correct total charge of the entire molecule $(-1)$. These systems are composed of a 1:3.4 $\mathrm{CholSO}_{4}$ : POPG ratio and generated in two dimensions. The box dimensions of the MD simulations are $(55.21 \times 55.21 \times$ $59.82 \AA)$ for the $55 \AA^{2} /$ lipid case, $(57.67 \times 57.67 \times 59.82$ $\AA)$ for the $60 \AA^{2} /$ lipid, $(60.02 \times 60.02 \times 59.82 \AA)$ for the $65 \AA^{2} /$ lipid, and $(62.28 \times 62.28 \times 59.82 \AA)$ for the $70 \AA^{2} /$ lipid. Electrostatic and Lennard-Jones interaction were considered with $12 \AA$ cutoffs and $10 \AA$ tapers. The switching is performed so that the force, not the potential, smoothly decays to zero within this $2 \AA$ tapering region. Each simulation consists of $0.5 \mathrm{~ns}$ equilibration at $300 \mathrm{~K}$ using Nose-Hoover thermostat NVT MD simulations with a relaxation time of $0.1 \mathrm{ps,}$ followed by $2.0 \mathrm{~ns}$ of production $N V T$ simulations for the molecular distribution analysis.

\section{Results and Discussion}

\section{Air-Liquid Interfacial Reaction of $\mathrm{CholSO}_{4}$ with $\mathrm{O}_{3}$}

The negative ion FIDI-MS spectra for ozonolysis of $\mathrm{CholSO}_{4}$ in a water/methanol (1:1 by volume) droplet are shown in Figure 1a. Prior to $\mathrm{O}_{3}$ application, singly deprotonated $\mathrm{CholSO}_{4}$, observed at $\mathrm{m} / z$ 465, is seen as a dominant species in the FIDI-MS spectrum. Highly abundant products resulting from ozonolysis of $\mathrm{CholSO}_{4}$ at the air-liquid interface appear as early as $5 \mathrm{~s}$ after exposing the 
(a)

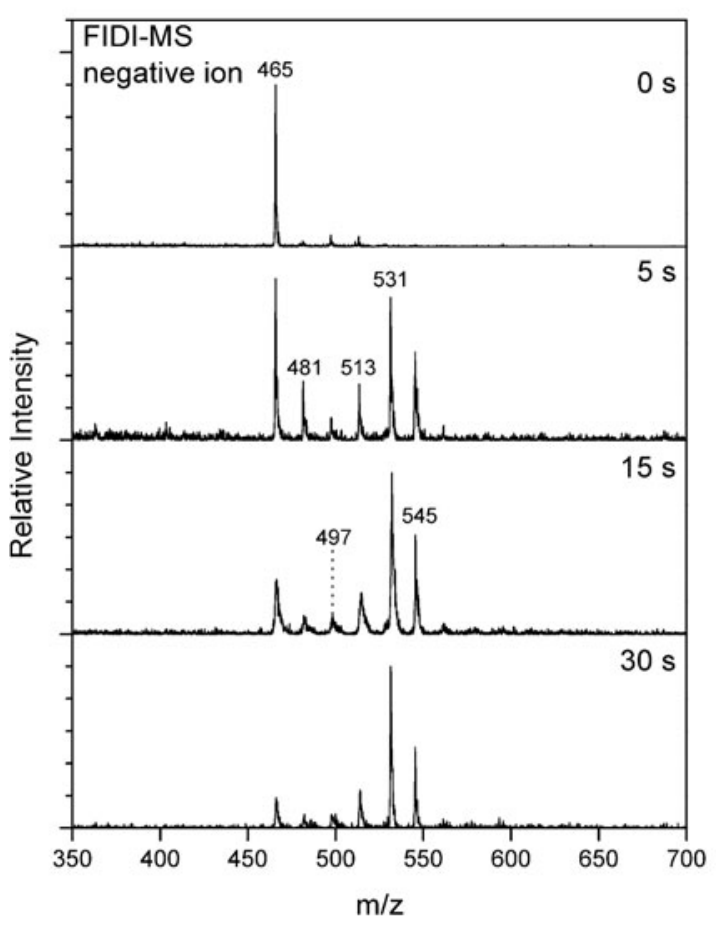

(b)

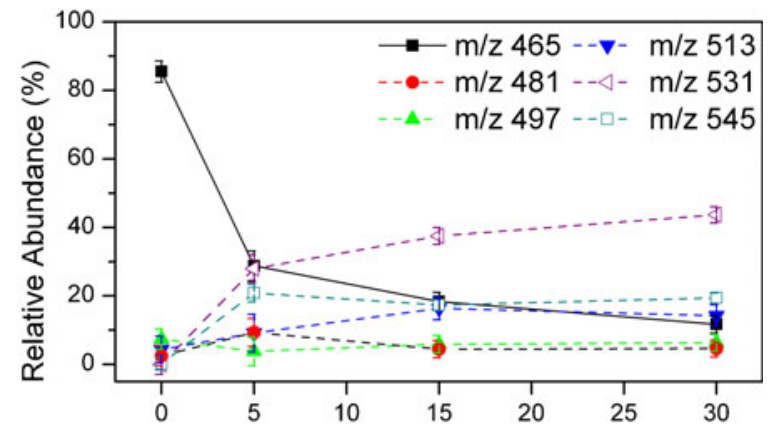

(c)

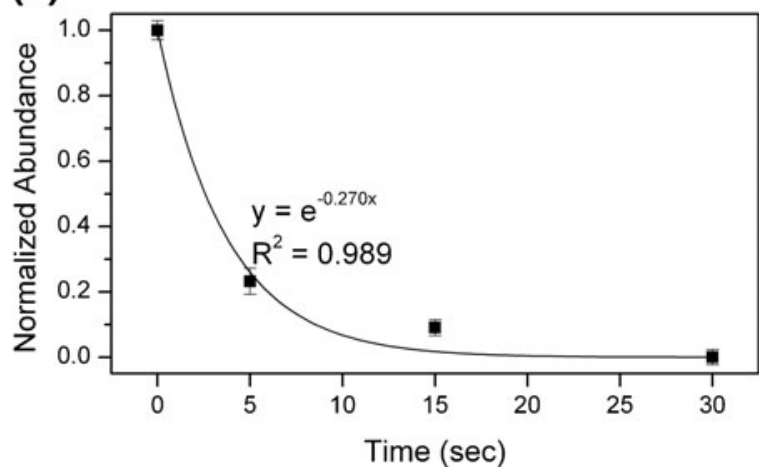

Figure 1. (a) Air-liquid interfacial reaction of $\mathrm{CholSO}_{4}$ with $\mathrm{O}_{3}$ as a function of time. The negative ion FIDI-MS spectrum of $\mathrm{CholSO}_{4}$ is dominated by the singly deprotonated $\mathrm{CholSO}_{4}$ peak at $\mathrm{m} / \mathrm{z} 465$ in the absence of ozone. The products appear after the droplet is exposed to $\mathrm{O}_{3}$ for $5 \mathrm{~s}$. After $15 \mathrm{~s}$ of ozone exposure, the FIDI-MS spectrum is dominated by oxidative products. Deprotonated HHP (product IV) and MHP (product V) products are observed at $\mathrm{m} / \mathrm{z} 531$ and 545 , respectively. Singly (product I), doubly (product II), and triply (product III) oxygenated products are shown at $\mathrm{m} / \mathrm{z} 481$, 497, and 513 , respectively. The structure of each product is shown in Scheme 2. (b) The relative abundance of the reactant and products of air-liquid interfacial ozonolysis of $\mathrm{CholSO}_{4}$ in the FIDI-MS spectra as a function of reaction time. (c) Plot of the abundance changes of $\mathrm{CholSO}_{4}$ in the ozonolysis at the air-liquid interface. Solid line is the exponential fit to determine the rate constant

droplet to $\mathrm{O}_{3}$. The products at $\mathrm{m} / \mathrm{z} 481,497$, and 513 correspond to singly $(+16)$, doubly $(+32)$, and triply $(+48)$ oxygenated $\mathrm{CholSO}_{4}$, respectively. Based on the previous studies of ozonolysis of Chol [25-29], singly, doubly, and triply oxygenated products are suggested as epoxide (product I, $m / z$ 481), dicarbonyl (product II, $m / z$ 497), and carbonyl-acid (product III, $\mathrm{m} / \mathrm{z}$ 513), respectively. Alternatively, the vinyl hydroperoxide may be generated for product III $[26,30]$. In addition, what we assume to be hydroxyhydroperoxide (HHP), methoxyhydroperoxide (MHP) products are observed at $m / z 531$ and 545, respectively [29, 31]. The proposed structures of products and reaction mechanisms are shown in Scheme 2. There are structural isomers and many reaction mechanisms may exit, but only some pathways of the observed products are summarized here. This putative analysis needs further validation using accurate mass measurement, which is beyond the scope of the present study.

The relative abundance of the reactant $\mathrm{CholSO}_{4}$ decreases dramatically after $15 \mathrm{~s}$ of exposure, and the FIDI-MS spectrum is dominated by ozonolysis products after $30 \mathrm{~s}$. The relative abundance changes of the reactant and products as a function of reaction time are found in Figure 1b. During the ozonolysis reaction, the ozone concentration is assumed to be constant. Then, the reaction rate of $\mathrm{CholSO}_{4}$ is expressed as

$$
-\frac{d\left[\mathrm{CholSO}_{4}\right]_{\text {surf }}}{d t}=k_{2}\left[\mathrm{CholSO}_{4}\right]_{\text {surf }, 0}
$$

where $k_{2}=k_{1}\left[\mathrm{O}_{3}\right]$ using the pseudo-first order kinetics. Solving Equation (1) gives

$$
\frac{\left[\mathrm{CholSO}_{4}\right]_{\text {surf }}}{\left[\mathrm{CholSO}_{4}\right]_{\text {surf }, 0}}=e^{-k_{2} t}
$$

The applied ozone concentration is $5 \times 10^{14}$ molecule $\mathrm{cm}^{-3}$ $(20 \mathrm{ppm})$. As seen in Figure 1c, $k_{2}=0.27 \mathrm{~s}^{-1}$ is obtained from $\mathrm{CholSO}_{4}$ abundance in FIDI-MS spectra. Then, secondorder rate constant, $k_{l}$, is determined as $5.4 \times 10^{-16} \mathrm{~cm}^{3}$ molecule $\mathrm{s}^{-1}$. The observed $\mathrm{CholSO}_{4}$ ozonolysis rate constant at the air-liquid interface is comparable to the ozonolysis rate constant of 1-oleoyl-2-palmitoyl-sn-phosphatidylcholin (OPPC) monolayer on $\mathrm{NaCl}\left(4.5 \times 10^{-16} \mathrm{~cm}^{3}\right.$ molecule $\mathrm{s}^{-1}$ ) [32]. Our previous study of POPG ozonolysis at the air-liquid interface $\left(7.4 \times 10^{-16} \mathrm{~cm}^{3}\right.$ molecule $\left.\mathrm{e}^{-1} \mathrm{~s}^{-1}\right)$ using FIDI-MS has also shown a good agreement with these values 


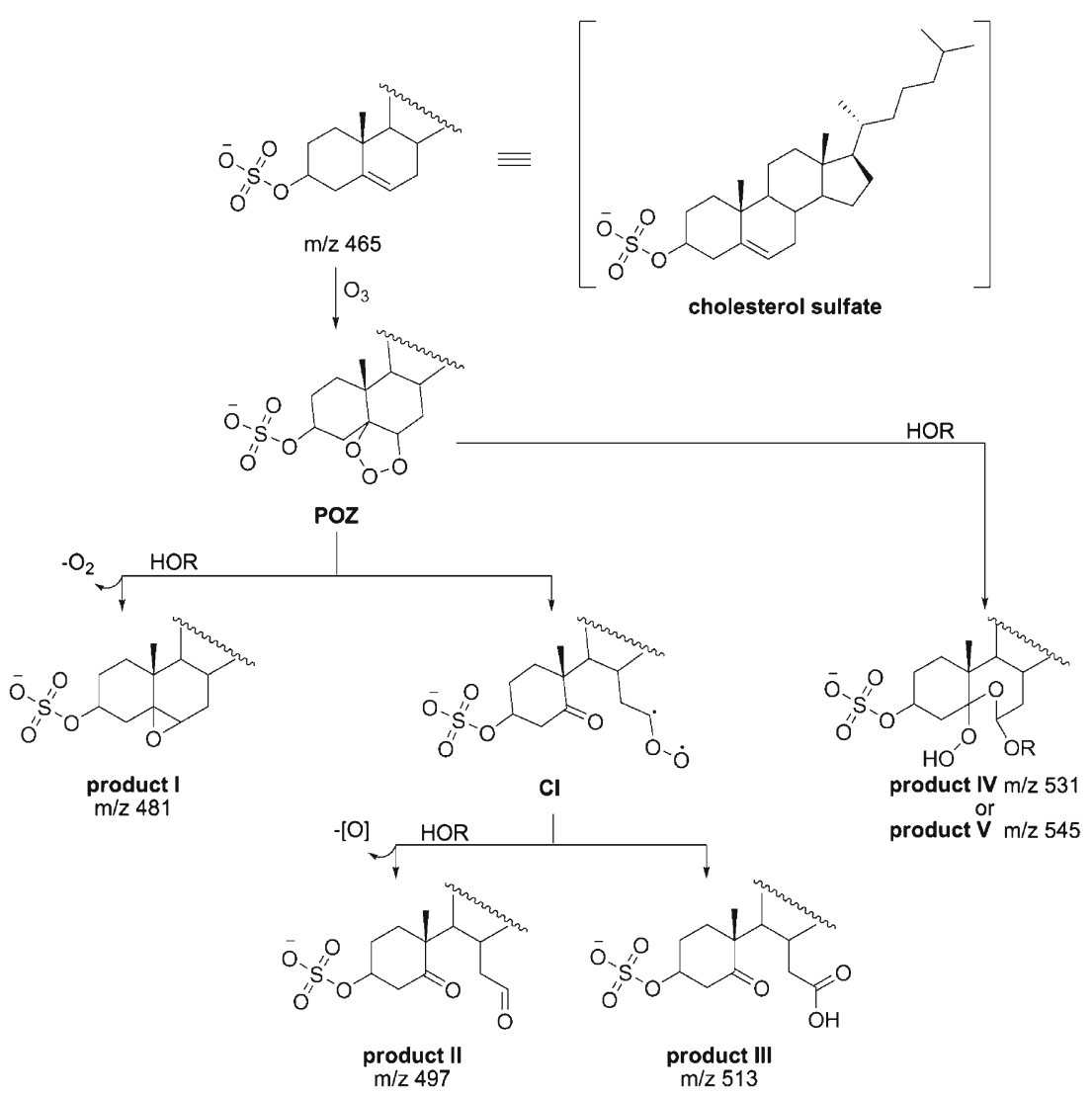

Scheme 2. Summary of heterogeneous oxidation of cholesterol sulfate by $\mathrm{O}_{3}$ at the air-liquid interface. $\mathrm{R}$ is $\mathrm{H}$ for water and $\mathrm{CH}_{3}$ for methanol. Proposed structures of products I, II, and III are adopted from Reference [25-29]. Proposed structures of products IV and V are adopted from Reference [29, 31]

[15]. The experimentally determined rate constant values are listed in Table 1.

It is notable that the oxygenated products I, II, and III appear at the beginning of the ozonolysis $\left(\mathrm{O}_{3}\right.$ exposure for $\left.5 \mathrm{~s}\right)$. However, as the droplet is exposed to $\mathrm{O}_{3}$ for $15 \mathrm{~s}$, a relatively low abundance of these products is observed in the FIDI-MS spectrum (Figure 1b). After $30 \mathrm{~s}$ exposure of $\mathrm{O}_{3}$ to the droplet, only a small amount of product III is observed along with highly abundant product IV and product $\mathrm{V}$. The air-liquid interfacial ozonolysis of $\mathrm{CholSO}_{4}$ yields $\mathrm{HHP}$ (product IV) and MHP (product V) products as the most abundant products. In order to yield HHP, a primary ozonide (POZ) reacts with a water molecule (Scheme 2) [32, 33]. However, this unstable product converts to an aldehyde product through a proton transfer with a water molecule in the bulk phase [33]. Abundant hydroperoxide products in the FIDI-MS spectra have been reported in our previous study of heterogeneous POPG ozonolysis [15]. These products are observed in the spectra due to low water abundance at the air-liquid interface. Similarly, the abundant HHP product from $\mathrm{CholSO}_{4}$ ozonolysis is attributed to a relatively low water density in the $\mathrm{CholSO}_{4}$ interfacial layer. At the air-water interface, Chol assembles in ordered crystalline structures up to three molecular layers thick $[34,35]$. Analysis using X-ray diffraction indicates that the Chol crystalline phase on the liquid surface comprises Chol monohydrate crystallites [35]. It is expected that $\mathrm{CholSO}_{4}$ interacts with water molecules to a greater extent than Chol due to its anionic sulfate head group. In contrast to the behavior of Chol with the hydroxyl group, solvation of the anionic sulfate group may prevent $\mathrm{CholSO}_{4}$ from forming multilayer crystalline hydrates at the air-liquid interface.

No dimeric or trimeric product is observed in the FIDIMS spectra. The study of Chol ozonolysis in a gas phase

Table 1. Experimentally Determined Ozonolysis Rate Constant Values of $\mathrm{CholSO}_{4}$ and POPG

\begin{tabular}{llcll}
\hline & \multicolumn{1}{c}{$\mathrm{CholSO}_{4}$} & $\mathrm{CholSO}_{4}$ (with POPG) & \multicolumn{1}{c}{ POPG } & POPG (with CholSO \\
\cline { 2 - 5 }$k_{2}{ }^{\text {a }}$ & $0.27 \pm 0.05$ & $0.18 \pm 0.03$ & $0.3708 \pm 0.0002$ & $0.30 \pm 0.03$ \\
$k_{1}{ }^{\mathrm{b}}$ & $(5.4 \pm 1.0) \times 10^{-16}$ & $(3.6 \pm 0.6) \times 10^{-16}$ & $(7.4 \pm 0.6) \times 10^{-16}$ & $(5.9 \pm 0.8) \times 10^{-16}$ \\
\hline
\end{tabular}

${ }^{\text {a }}$ Pseudo-first order rate constant $\left(\mathrm{s}^{-1}\right)$

${ }^{\mathrm{b}} \mathrm{Second}$ order rate constant $\left(\mathrm{cm}^{3}\right.$ molecule $\left.\mathrm{s}^{-1} \mathrm{~s}^{-1}\right)$ 
aerosol reported bound multimeric products, which are formed through aggregated gas phase clusters [26]. The absence of multimeric product from heterogeneous ozonolysis suggests that $\mathrm{CholSO}_{4}$ molecules do not aggregate and form well-oriented layer structures on the surface of the droplet.

\section{Solution and Solid Phase Reaction of $\mathrm{CholSO}_{4}$ with $\mathrm{O}_{3}$}

Solution and solid phase reactions with $\mathrm{O}_{3}$ have been performed in order to understand the unique characteristics of air-liquid interfacial ozonolysis of $\mathrm{CholSO}_{4} \cdot \mathrm{O}_{3}$ is bubbled into the $\mathrm{CholSO}_{4}$ solution and applied to a dried $\mathrm{CholSO}_{4}$ crystal film for solution-phase and solid-phase reactions, respectively. Figure 2 shows ESI-MS spectra of ozonolysis products of $\mathrm{CholSO}_{4}$ after bubbling of $20 \mathrm{ppm}$ $\mathrm{O}_{3}$ into the $200 \mu \mathrm{L} \mathrm{CholSO}_{4}$ solution (Figure 2a) and after applying $20 \mathrm{ppm} \mathrm{O}_{3}$ to a dried $\mathrm{CholSO}_{4}$ film $(140 \mu \mathrm{g}$, Figure 2b).

All products observed from the air-liquid interfacial ozonolysis of $\mathrm{CholSO}_{4}$ (Figure 1a) are observed from the solution phase ozonolysis with different product distribution (Figure 2a). The solution phase ozonolysis of $\mathrm{CholSO}_{4}$ also yields HHP (product IV, $\mathrm{m} / \mathrm{z}$ 531) and MHP (product V, $\mathrm{m} / \mathrm{z}$ $545)$ products as the most abundant products. The significant difference compared with air-liquid interfacial ozonolysis is observed from the relative abundance of three oxygenated products (products I, II, and III). After bubbling of $\mathrm{O}_{3}$ for $60 \mathrm{~s}$, product I $(\mathrm{m} / \mathrm{z} 481)$ appears as the most abundant product in the ESI-MS spectrum while product III at $\mathrm{m} / \mathrm{z} 513$ is shown as the least abundant product among products I, II, and III.

The solid phase reaction yields different products except for triply oxygenated product (product III) at $\mathrm{m} / \mathrm{z} 513$ (Figure 2b). Singly and doubly oxygenated products are not observed at $\mathrm{m} / \mathrm{z} 481$ and 497, respectively, in the ESIMS spectrum after applying $\mathrm{O}_{3}$ to a dried $\mathrm{CholSO}_{4}$ film for $12 \mathrm{~h}$. As well, hydroperoxide products $(\mathrm{m} / \mathrm{z} 531$ and 545) are not observed. Instead, a diol $(+34)$ product is observed at $\mathrm{m} / \mathrm{z}$ 499. The absence of product I (epoxide), product II (dicarbonyl), and hydroperoxide (products IV and V) products from ozonolysis of the $\mathrm{CholSO}_{4}$ film implies that formation of these products requires a wet environment. (a)

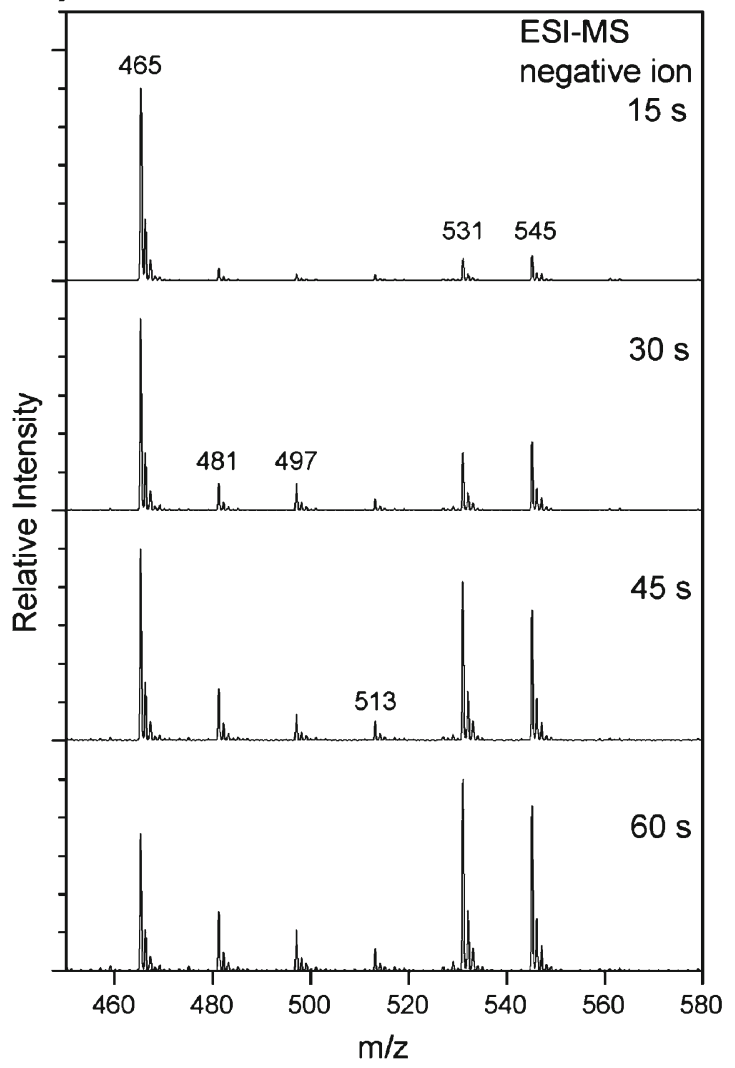

(b)

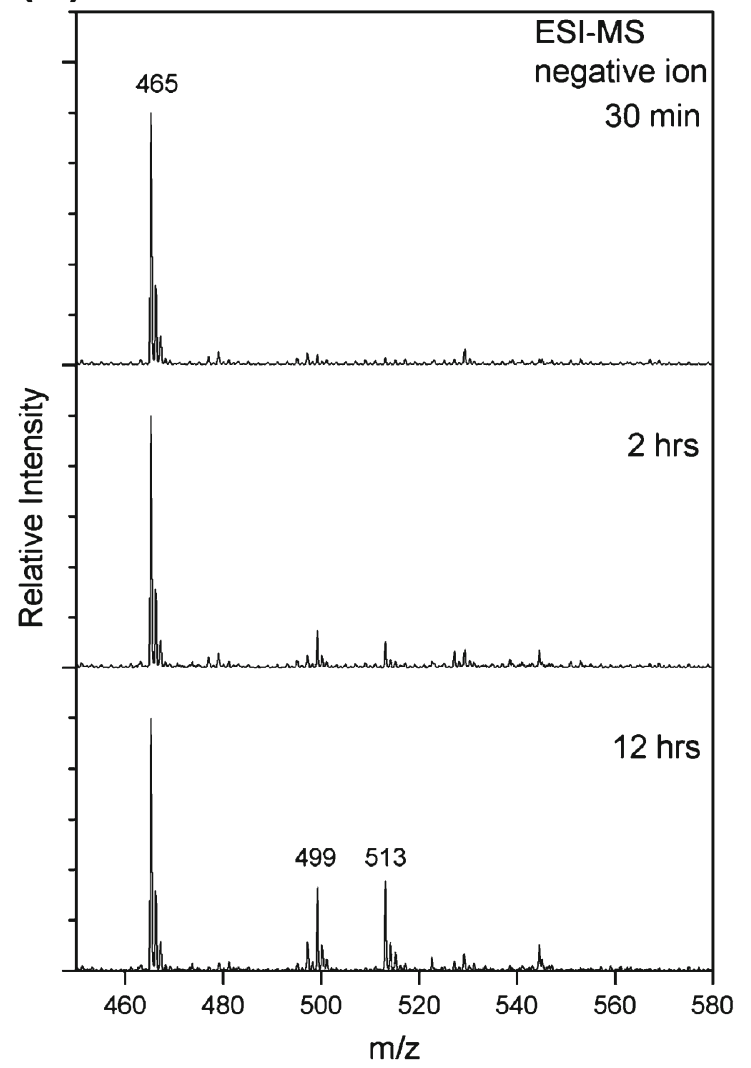

Figure 2. (a) ESI-MS spectra of the oxidized products of $\mathrm{CholSO}_{4}$ from solution phase $\mathrm{O}_{3}$ reaction as a function of time. Singly deprotonated $\mathrm{CholSO}_{4}$ is observed at $\mathrm{m} / \mathrm{z} 465$ along with deprotonated singly, doubly, and triply oxygenated products at $\mathrm{m} / \mathrm{z}$ 481, 497, and 513, respectively. HHP and MHP products are observed at $\mathrm{m} / \mathrm{z} 531$ and 545, respectively. (b) ESI-MS spectra of the oxidized products of $\mathrm{CholSO}_{4}$ from solid-phase $\mathrm{O}_{3}$ reaction as a function of time. The singly deprotonated $\mathrm{CholSO}_{4}$ is observed at $\mathrm{m} / \mathrm{z} 465$. The products at $\mathrm{m} / \mathrm{z} 499$ and 513 correspond to diol product and carbonyl-acid product. The ion at $\mathrm{m} / \mathrm{z}$ 544.5 is unknown cluster ion comprise $\sim 141.8$ mass unit monomer and singly charged anion with $119 \mathrm{~m} / \mathrm{z}$ 


\section{The Role of Water Molecules in Ozonolysis of $\mathrm{CholSO}_{4}$}

Previous studies of the ozonolysis of Chol have reported that the epoxide product is observed only in a polar solvent [25, 36]. Formation of this product does not occur in an aprotic solvent [25]. The epoxide product is also observed from free-radical peroxidation processes [36]. Once $\mathrm{O}_{3}$ is dissolved in a polar solvent (i.e., water), unstable $\mathrm{O}_{3}$ rapidly forms secondary reactive oxygen species (ROS), including $\mathrm{OH}$ radical [37]. This implies that the formation of an epoxide product (product I in Scheme 2) involves either an ozonolysis process aided by polar solvent or oxidation of $\mathrm{CholSO}_{4}$ with a secondary ROS [38]. However, $\mathrm{O}_{3}$ has limited solubility in an aqueous solution, with a very low Henry's law constant $(0.011 \mathrm{M} / \mathrm{atm})$ [39]. This results in a very limited number of ROS in solution to produce the abundant epoxide product. This suggests that interactions of a Criegee intermediate $(\mathrm{CI})$ or a primary ozonide $(\mathrm{POZ})$ with solvent molecules (water or methanol) would be a responsible for the formation of product I $(\mathrm{m} / \mathrm{z} 481$ in Figures 1a and 2a) from the ozonolysis of $\mathrm{CholSO}_{4}$. The role of solvent molecules to yield the dicarbonyl product (product II in Scheme 2, m/z 497 in Figure 1a) is unclear, but dicarbonyl product is known to be highly abundant interfacial ozonolysis product of Chol and even suggested as a biomarker for ozone exposure of lung, where constant heterogeneous reactions occur $[27,28]$. The carbonyl-acid product (product III) at $m / z 513$ is the least abundant product from solution phase ozonolysis (Figure 2a) while it is the most abundant product from solid phase ozonolysis (Figure 2b). The formation of a carbonyl-acid product does not require a solvent molecule. Thus, this product is highly abundant with a dry environment and observed in much lower yields in a wet environment.

As seen in Figure 1b, the abundance of the epoxide product (product I, $\mathrm{m} / \mathrm{z} 481$ ) is comparable to the carbonylacid product (product III, $\mathrm{m} / \mathrm{z}$ 513) after the droplet is exposed to $\mathrm{O}_{3}$ for $5 \mathrm{~s}$. However, its abundance decreases dramatically relative to other products after exposure of the droplet to $\mathrm{O}_{3}$ for $15 \mathrm{~s}$. This indicates an important characteristic of the air-liquid interface. As discussed earlier, low water density in the $\mathrm{CholSO}_{4}$ layer is expected at the air-liquid interface. The limited number of water molecules around the double bond of $\mathrm{CholSO}_{4}$ is consumed to form HHP (product IV). This results in the low yield of the epoxide product via ozonolysis of $\mathrm{CholSO}_{4}$ at the air-liquid interface. A similar phenomenon is observed from the previous study of the ozonolysis of POPG at the air-liquid interface [15]. The secondary ozonide of POPG (POPG$\mathrm{SOZ}$ ), which forms only under an anhydrous environment, starts building up after the limited water molecules are depleted around the double bond of POPG. The relatively low abundance of the product II is also a consequence of the limited number of water molecules around the double bond of $\mathrm{CholSO}_{4}$. Solution phase ozonolysis of $\mathrm{CholSO}_{4}$ shows a higher abundance of product II $(\mathrm{m} / \mathrm{z} 497)$ than product III $(\mathrm{m} / \mathrm{z}$ 513), while only the latter is observed under a dry environment (Figure 2). Once the $\mathrm{CI}$ forms from the $\mathrm{POZ}$ of $\mathrm{CholSO}_{4}$ (Scheme 2), after the limited water molecules are depleted, the carbonyl-acid product is preferentially formed at the air-liquid interface.

\section{Air-Liquid Interfacial Reaction of a $\mathrm{CholSO}_{4}$ and POPG Mixture with $\mathrm{O}_{3}$}

Negative ion FIDI-MS spectra for the time resolved ozonolysis of an equimolar mixture of $\mathrm{CholSO}_{4}$ and POPG at the air-liquid interface are shown in Figure 3a. Singly deprotonated $\mathrm{CholSO}_{4}$ and POPG, are observed at $\mathrm{m} / z 465$ and 747 , respectively. In the FIDI-MS spectrum, POPG exhibits higher abundance than $\mathrm{CholSO}_{4}$ in the spectrum and the abundance of $\mathrm{CholSO}_{4}$ is $\sim 76 \%$ of the POPG abundance.

Our recent study of the ozonolysis of POPG at the airliquid interface has shown that products of POPG appear as early as $5 \mathrm{~s}$ after exposing the droplet to $20 \mathrm{ppm} \mathrm{O}_{3}$ [15]. All products of POPG previously observed, including aldehyde (m/z 637), carboxylic acid ( $\mathrm{m} / \mathrm{z} 653)$, peroxic acid $(\mathrm{m} / \mathrm{z} 669)$, POPG hydroxyhydroperoxide (POPG-HHP, $m / z$ 671), and POPG methoxyhydroperoxide (POPG-MHP, $m / z$ 685), are also observed from the ozonolysis of POPG in the mixture with $\mathrm{CholSO}_{4}$ (Scheme 3). The POPG-SOZ at $\mathrm{m} / \mathrm{z} 795$ shows up after exposing droplet to $\mathrm{O}_{3}$ for $15 \mathrm{~s}$ before POPG is depleted. In the mixture, only HHP $(\mathrm{m} / \mathrm{z} 531)$ and MHP $(\mathrm{m} / \mathrm{z} 545)$ products are observed in significant yield in the FIDI-MS data.

Ozonolysis rates of $\mathrm{CholSO}_{4}$ and POPG in the mixture decrease compared with when each molecule is present alone (Figure $3 \mathrm{~b}$ ). The observed rate constant values, $k_{1}$, of $\mathrm{CholSO}_{4}$ and POPG are $3.6 \times 10^{-16} \mathrm{~cm}^{3}$ molecule ${ }^{-1} \mathrm{~s}^{-1}$ and $5.9 \times 10^{-16} \mathrm{~cm}^{3}$ molecule ${ }^{-1} \mathrm{~s}^{-1}$, respectively, in a mixture (Table 1). The slower reaction rates of both $\mathrm{CholSO}_{4}$ and POPG suggest that $\mathrm{CholSO}_{4}$ is present with POPG as a wellmixed interfacial layer supporting the previous study of its miscibility to liquid extended layers [40]. Our fluorescence microscopy observation of the mixture of $\mathrm{CholSO}_{4}$ (with $0.5 \mathrm{~mol}_{0}$ 25-NBD $\mathrm{CholSO}_{4}$ ) and POPG at the air-liquid interface also supports this showing homogeneous one phase image (Figure S1 in the Supplemental Information). Overall, the POPG ozonolysis rate decreases by $20 \%$ and $\mathrm{CholSO}_{4}$ ozonolysis rate decreases by $34 \%$ in a mixed layer (Figure 3b) [15]. As discussed earlier, air-liquid interfacial ozonolysis rate constants of both $\mathrm{CholSO}_{4}\left(5.4 \times 10^{-16} \mathrm{~cm}^{3}\right.$ molecule $\left.{ }^{-1} \mathrm{~s}^{-1}\right)$ and POPG $\left(7.4 \times 10^{-16} \mathrm{~cm}^{3}\right.$ molecule $\left.{ }^{-1} \mathrm{~s}^{-1}\right)$ are comparable. In addition, the ozone concentration is assumed to be constant during the ozonolysis. The observed time delay for overall ozonolysis of POPG and $\mathrm{CholSO}_{4}$ implies that double bonds of POPG and $\mathrm{CholSO}_{4}$ are more shielded by additional hydrocarbons from each other at the air-liquid interface. Slightly longer delay for the ozonolysis of $\mathrm{CholSO}_{4}$ indicates that shielding effect of POPG acyl chain to the double bond of $\mathrm{CholSO}_{4}$ is slightly higher 
(a)

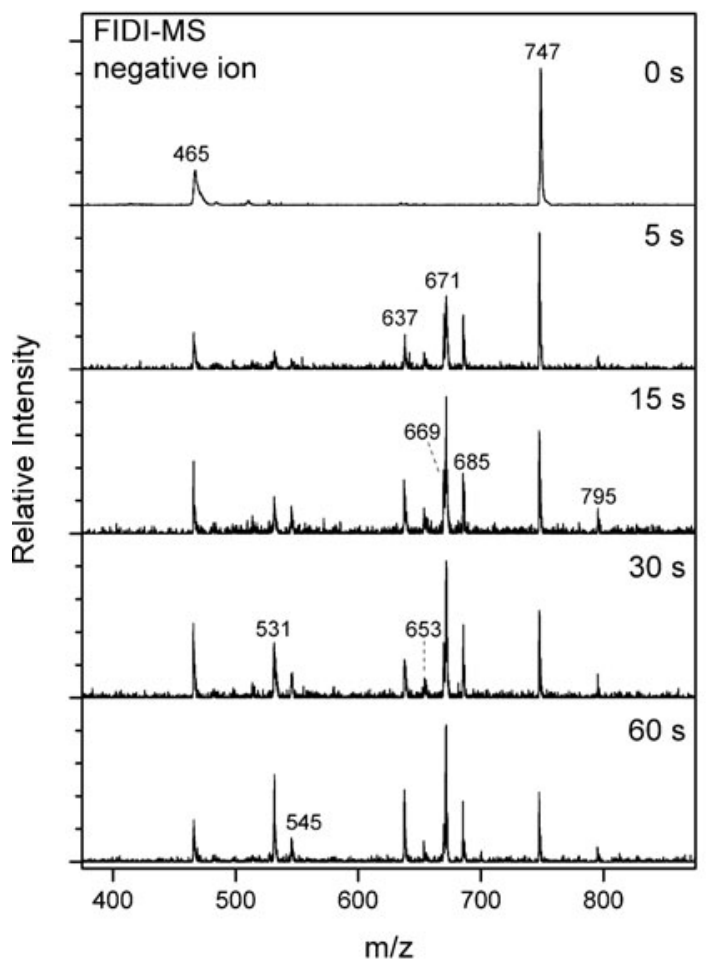

(b)

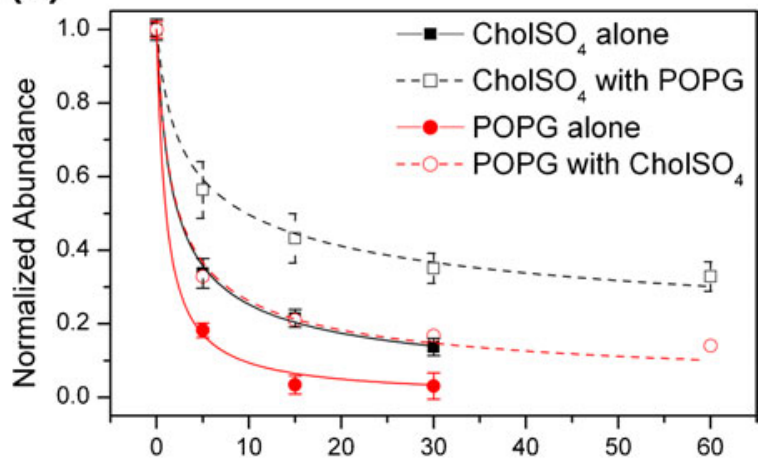

(c)

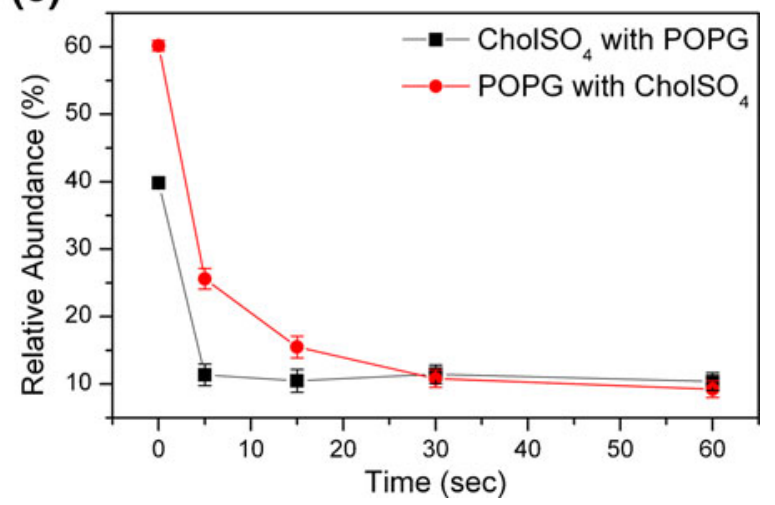

Figure 3. (a) Heterogeneous reaction of a 1:1 mixture of $\mathrm{CholSO}_{4}$ and $\mathrm{POPG}$ with $\mathrm{O}_{3}$ as a function of time. In the absence of ozone, singly deprotonated POPG and $\mathrm{CholSO}_{4}$ peaks are observed at $\mathrm{m} / \mathrm{z} 747$ and 465 , respectively. The oxidation products of POPG and $\mathrm{CholSO}_{4}$ are observed after $5 \mathrm{~s}$ of $\mathrm{O}_{3}$ exposure. The structure of each product from POPG is shown in $\mathrm{Scheme} 3$. (b) Plot of the abundance changes of $\mathrm{CholSO}_{4}$ (black square) and POPG (red circle) in the ozonolysis at the air-liquid interface. The abundance changes of $\mathrm{CholSO}_{4}$ and POPG when each molecule is present alone on the droplet are shown as solid square and circle, respectively. The abundance changes of POPG is adopted from Ref [15]. The abundance changes of $\mathrm{CholSO}_{4}$ and POPG in a mixture layer are shown as empty square and circle, respectively. Solid lines are the exponential fits to determine the rate constants when each molecule is present alone and dashed lines are the exponential fits when they are in a mixture layer. (c) The relative abundance of the $\mathrm{CholSO}_{4}$ (black solid square) and POPG (red solid circle) during the ozonolysis at the air-liquid interface in the FIDI-MS spectra as a function of reaction time

compared with the shielding effect of $\mathrm{CholSO}_{4}$ to the POPG double bond. The relative abundance of $\mathrm{CholSO}_{4}$ increases after $30 \mathrm{~s}$ of $\mathrm{O}_{3}$ exposure (Figure $3 \mathrm{c}$ ). Slightly more reactive
POPG yields more hydrophilic ozonolysis products, which diffuse into the aqueous droplet [15] increasing relative abundance of $\mathrm{CholSO}_{4}$ in the interfacial surfactant layer.

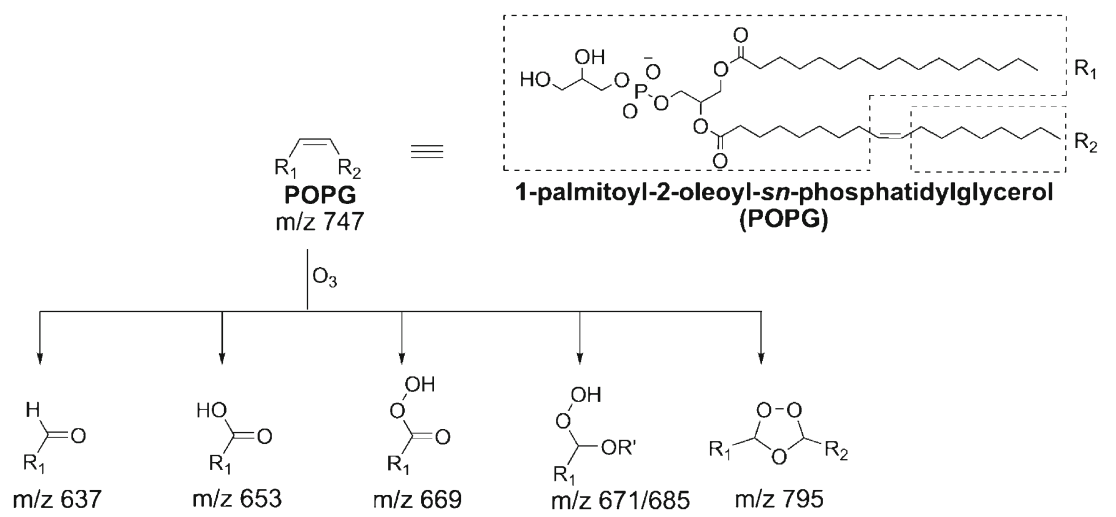

Scheme 3. Summary of the observed products from heterogeneous oxidation of POPG by $\mathrm{O}_{3}$ at the air-liquid interface. $\mathrm{R}^{\prime}$ is $\mathrm{H}$ for water and $\mathrm{CH}_{3}$ for methanol. Product structures are adopted from Reference [15] 
It is notable that the POPG-SOZ is formed before POPG is depleted in the spectrum. Previous study of the heterogeneous ozonolysis of POPG has shown that POPG-SOZ starts building up after POPG is depleted on the surface of the droplet [15]. The early appearance of POPG-SOZ suggests rapid depletion of the limited number of water molecules in the hydrophobic portion of the lipid layer due to the co-consumption of water molecules with $\mathrm{CholSO}_{4}$.

\section{Interactions of $\mathrm{CholSO}_{4}$ and POPG Double Bonds with Water Molecules at the Air-Liquid Interface}

MD simulations for the mixture monolayer of $\mathrm{CholSO}_{4}$ and POPG on a water box are performed for 2.0 ns. Four different surface densities $\left(55,60,65\right.$, and $70 \AA^{2} /$ lipid $)$ of $\mathrm{CholSO}_{4}$ and a POPG mixture monolayer are used for the simulations. These surface densities are reported as a reasonable density range of a lipid monolayer at the airliquid interface [41-43]. The ratio between $\mathrm{CholSO}_{4}$ and POPG in the monolayer is set to be 1:3.4. In order to investigate the effect of $\mathrm{CholSO}_{4}$ abundance in the monolayer, additional $\mathrm{MD}$ simulation for the mixture monolayer of $\mathrm{CholSO}_{4}$ and POPG with ratio of 1:1 is also performed for $2.0 \mathrm{~ns}$ using the surface density of $60 \AA^{2} /$ lipid.
Figure 4a shows a final snapshot of the MD simulation, when the ratio of $\mathrm{CholSO}_{4}$ to POPG is 1:3.4, for 2.0 ns with the surface density of $60 \AA^{2} /$ lipid as a representative case. Figure $4 \mathrm{~b}$ shows the atomic density profiles of oxygen atoms of water molecules, $\mathrm{sp}^{2}$ carbons (double bond) of lipid acyl chains along $\pm \Delta z$, which is the z-direction location relative to the averaged position of the POPG phosphorous atom resulting from the simulation using 1:3.4 $\mathrm{CholSO}_{4}$ and POPG. The interaction between lipid functional groups and water can be estimated from the density of water molecule oxygen atom at the z-direction location of the lipid functional group. The water density around the double bonds of $\mathrm{CholSO}_{4}$ is $\sim 0.0036$ atom $/ \AA^{3}$. Compared with the bulk phase where water density is $\sim 0.034$ atom $/ \AA^{3}$, a limited number of water molecules are present around the double bond of $\mathrm{CholSO}_{4}$ to be involved when ozone reacts with the double bond of $\mathrm{CholSO}_{4}$. As discussed earlier, this limited number of water molecules is mainly consumed to form HHP.

Based on the observed competitive reactivity of $\mathrm{CholSO}_{4}$ and POPG with $\mathrm{O}_{3}$ (Figure 3a), we have suggested that these molecules are co-located at the interface. Our interpretation is further supported by the MD simulations of a mixture monolayer of $\mathrm{CholSO}_{4}$ and POPG. Overall, POPG and $\mathrm{CholSO}_{4}$ show similar medial thickness on (a)

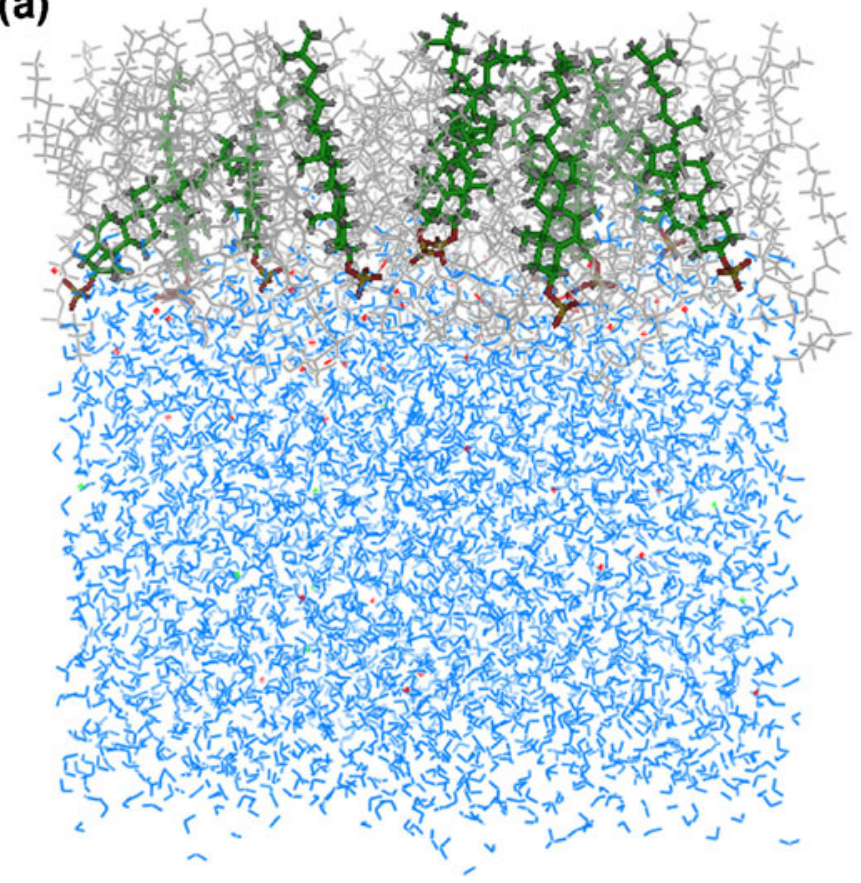

(b)
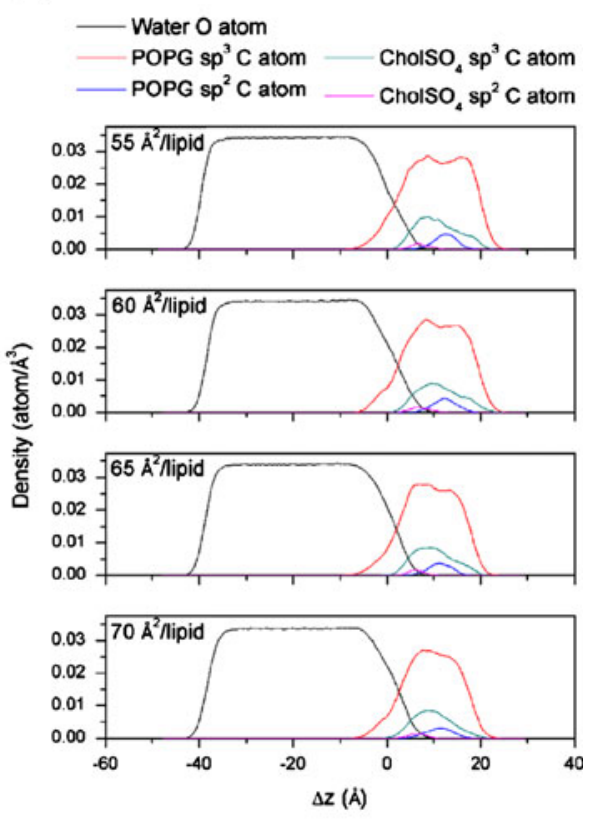

Figure 4. (a) Final snapshot after 2.0 ns of MD simulation of a mixture of $\mathrm{CholSO}_{4}$ and POPG monolayer at $60 \AA^{2} /$ lipid. $\mathrm{CholSO}_{4}$ (displayed as tube) is shown in olive (tail) and burgundy (head). POPG, water molecules, and sodium ions are shown in gray, blue, and purple, respectively. (b) Atomic density profiles of a mixture of $\mathrm{CholSO}_{4}$ and POPG monolayer systems as a function of $\Delta z$, where the averaged position of the POPG phosphorous atom at the air-liquid interface is 0 (negative values are toward the water layer, and positive values are toward the lipid). The lipid surface densities are $55 \AA^{2} /$ lipid, $60 \AA^{2} /$ lipid, $65 \AA^{2} /$ lipid, and $70 \AA^{2}$ /lipid. Black solid lines denote the density profiles of oxygen atoms of water molecules. Red solid lines denote that of $\mathrm{sp}^{3}$ carbons of POPG acyl chains, and blue solid lines denote that of $\mathrm{sp}^{2}$ carbons of POPG acyl chains. Olive and magenta solid lines denote density profiles of $\mathrm{sp}^{3}$ and $\mathrm{sp}^{2}$ carbons of $\mathrm{CholSO}_{4}$, respectively 
the surface of water. In other words, they are co-located in the lipid monolayer. When the lipid monolayer has a $60 \AA^{2} /$ lipid surface density, hydrophobic carbons of both lipids stand up to $\sim 14 \AA$. Although the overall dimension of POPG exceeds those of $\mathrm{CholSO}_{4}$, the hydrophobic portions of both molecules have a comparable extent (Scheme 1). As seen in Figure 5a, the sulfate group of $\mathrm{CholSO}_{4}$ directly interacts with as many as eight water molecules via ionic-hydrogen bonds. These strong interactions of the sulfate group with water molecules and the rigid ring structures of $\mathrm{CholSO}_{4}$ induce high tilt angle $\left(67^{\circ}\right)$ from the surface of the water. Therefore, $\mathrm{CholSO}_{4}$ and POPG form a well-mixed interfacial layer on the surface of the water. It is noteworthy that compared with the $\mathrm{sp}^{2}$ carbons of POPG, $\mathrm{sp}^{2}$ carbons of $\mathrm{CholSO}_{4}$ are located only $\sim 4.4 \AA$ closer to the water surface (Figure $5 b$ ).

To understand the observed time delay for overall ozonolysis of both POPG and $\mathrm{CholSO}_{4}$ in the mixed lipid layer, we have calculated the number of $\mathrm{sp}^{3}$ carbons from the surface of surfactant layer to the locations of double bonds, from the atomic density profiles. The calculated number of $\mathrm{sp}^{3}$ carbons per $\mathrm{sp}^{2}$ carbon in this region, using a surface density of $60 \AA^{2} /$ lipid, is summarized in Table 2. When only $\mathrm{CholSO}_{4}$ and
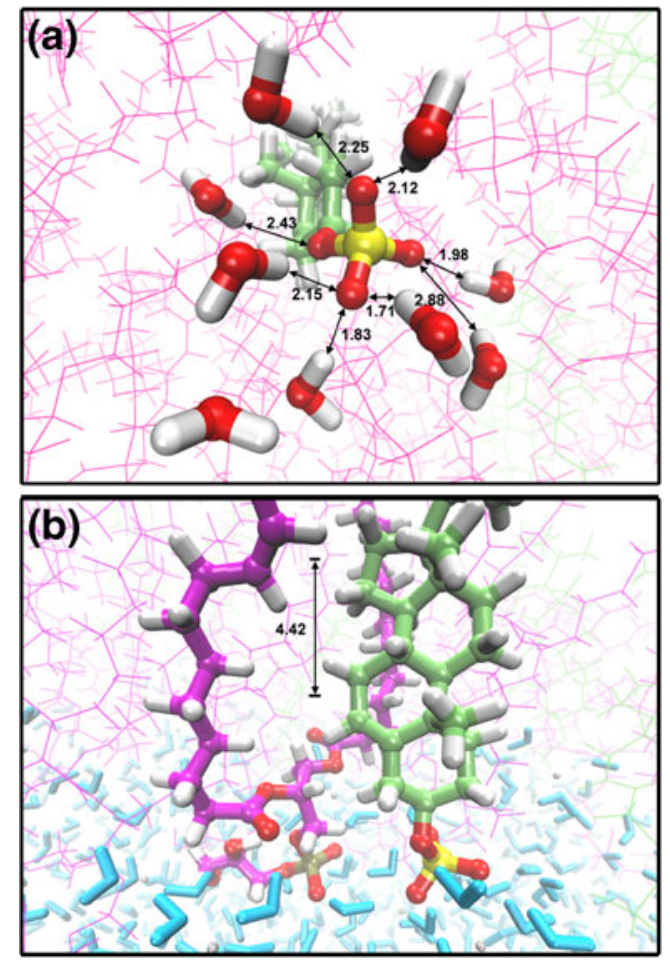

Figure 5. Final snapshot after $2.0 \mathrm{~ns}$ of MD simulation of a mixture of $\mathrm{CholSO}_{4}$ (lime) and POPG (magenta) monolayer at $60 \AA^{2} /$ lipid. (a) Sulfate group of $\mathrm{CholSO}_{4}$ is interacting with eight water molecules. (b) The $\mathrm{sp}^{2}$ carbons of $\mathrm{CholSO}_{4}$ are located $\sim 4.4 \AA$ below $\mathrm{sp}^{2}$ carbons of POPG. The calculated distances between atoms are indicated as Ångström
Table 2. The Number of $\mathrm{sp}^{3}$ Carbons per $\mathrm{sp}^{2}$ Carbon Calculated from the Surface of Surfactant Layer to the Locations of Double Bonds using a Surface Density of $60 \AA^{2} /$ lipid

\begin{tabular}{|c|c|c|}
\hline \multirow[t]{2}{*}{$\mathrm{CholSO}_{4}: \mathrm{POPG}$} & \multicolumn{2}{|c|}{$\mathrm{sp}^{3} / \mathrm{sp}^{2}$} \\
\hline & $\mathrm{CholSO}_{4}$ & POPG \\
\hline $1: 3.4$ & 70.4 & 18.6 \\
\hline $1: 1$ & 31.3 & 29.5 \\
\hline 1:0 ( $\mathrm{CholSO}_{4}$ only $)$ & 10.8 & - \\
\hline $0: 1$ (POPG only) & - & 15.8 \\
\hline
\end{tabular}

POPG are present on the surface of water, 10.8 and 15.8 $\mathrm{sp}^{3}$ carbons surround each $\mathrm{sp}^{2}$ carbon, respectively, and are located in the interior of the hydrophobic carbon layer. For each $\mathrm{sp}^{2}$ carbon of $\mathrm{CholSO}_{4}$ and POPG, the number of $\mathrm{sp}^{3}$ carbons in the mixed lipid layer increases to 70.4 and 18.6, respectively, when the ratio of $\mathrm{CholSO}_{4}$ to POPG is $1: 3.4$. In the case where the amount of $\mathrm{CholSO}_{4}$ is comparable to POPG in the monolayer (i.e., 1:1), our MD simulation shows each $\mathrm{sp}^{2}$ carbon of $\mathrm{CholSO}_{4}$ is surrounded by 31.3 and $29.5 \mathrm{sp}^{3}$ carbons in the monolayer, respectively (Figure S2 in the Supplemental Information). This strongly indicates that an increasing number of hydrocarbon chains eclipse the double bonds of $\mathrm{CholSO}_{4}$ and POPG as $\mathrm{O}_{3}$ diffuses into the mixed lipid monolayer. As a result, both POPG and $\mathrm{CholSO}_{4}$ ozonolysis is delayed in a mixed interfacial layer, as we observed from the experiments using FIDIMS (Figure 3).

\section{Conclusions}

We have utilized the FIDI-MS technique to examine the effect of environmental exposures on the surfactant layer at the air-liquid interface. This study provides details for the reaction of $\mathrm{CholSO}_{4}$ with $\mathrm{O}_{3}$ to understand the unique chemistry of this molecule at an air-liquid interface. Time-resolved studies of ozonolysis of $\mathrm{CholSO}_{4}$ at the air-liquid interface reveal that a limited amount of water around double bonds of $\mathrm{CholSO}_{4}$ plays an important role in yielding oxygenated products. The epoxide and dicarbonyl products are observed only when water molecules are present around double bonds of $\mathrm{CholSO}_{4}$. Competitive oxidation of $\mathrm{CholSO}_{4}$ and POPG at the air-liquid interface suggests that both lipids form a mixed interfacial layer when they are present together in a lipid surfactant layer. In a mixed layer, the double bonds of $\mathrm{CholSO}_{4}$ and POPG are more shielded by additional hydrocarbons from each other resulting in a time delay for the ozonolysis of both molecules. MD simulations of a mixed interfacial monolayer of $\mathrm{CholSO}_{4}$ and POPG provide a detailed picture of the interactions between POPG, CholSO ${ }_{4}$, and water molecules in the interfacial region. In these simulations, the location and orientation of $\mathrm{CholSO}_{4}$ relative to POPG provide a rationalization for the experimental observations. 


\section{Acknowledgment}

The authors acknowledge financial supported for this work by Basic Science Research Program (to H.I.K.; grant no. 2010-0021508) and by WCU Program (to S.M.C. and Y.M. R.; grant no. R32-2008-000-10180-0) through the National Research Foundation of Korea funded by the Ministry of Education, Science, and Technology. The authors also acknowledge financial support provided by the Beckman Institute Mass Spectrometry Resource Center and National Science Foundation of the United States under grant no. CHE-0416381 (to J.L.B., PI).

\section{References}

1. Strott, C.A., Higashi, Y.: Cholesterol sulfate in human physiology: What's it all about? J. Lipid Res. 44, 1268-1278 (2003)

2. Bleau, G., Bodley, F.H., Longpré, J., Chapdelaine, A., Roberts, K.D.: Cholesterol sulfate. I. Occurrence and possible biological functions as an amphipathic lipid in the membrane of the human erythrocyte. Biochim. Biophys. Acta-Biomembr. 352, 1-9 (1974)

3. Langlais, J., Zollinger, M., Plante, L., Chapdelaine, A., Bleau, G., Roberts, K.D.: Localization of cholesteryl sulfate in human spermatozoa in support of a hypothesis for the mechanism of capacitation. Proc. Natl. Acad. Sci. U.S.A. 78, 7266-7270 (1981)

4. Eberlin, L.S., Dill, A.L., Costa, A.B., Ifa, D.R., Cheng, L., Masterson, T., Koch, M., Ratliff, T.L., Cooks, R.G.: Cholesterol sulfate imaging in human prostate cancer tissue by desorption electrospray ionization mass spectrometry. Anal. Chem. 82, 3430-3434 (2010)

5. Smondyrev, A.M., Berkowitz, M.L.: Molecular dynamics simulation of dipalmitoylphosphatidylcholine membrane with cholesterol sulfate. Biophys. J. 78, 1672-1680 (2000)

6. Faure, C., Dufourc, E.J.: The thickness of cholesterol sulfate-containing membranes depends upon hydration. Biochim. Biophys. Acta-Biomembr. 1330, 248-253 (1997)

7. Faure, C., Tranchant, J.F., Dufourc, E.J.: Comparative effects of cholesterol and cholesterol sulfate on hydration and ordering of dimyristoylphosphatidylcholine membranes. Biophys. J. 70, 13801390 (1996)

8. Le Grimellec, C., Daigneault, A., Bleau, G., Roberts, K.: Cholesteryl sulfate-phosphatidylcholine interactions. Lipids 19, 474-477 (1984)

9. Rog, T., Pasenkiewicz-Gierula, M., Vattulainen, I., Karttunen, M.: Ordering effects of cholesterol and its analogues. Biochim. Biophys. Acta-Biomembr. 1788, 97-121 (2009)

10. Martinez-Seara, H., Rog, T., Karttunen, M., Reigada, R., Vattulainen, I.: Influence of cis double-bond parametrization on lipid membrane properties: How seemingly insignificant details in force-field change even qualitative trends. J. Chem. Phys. 129, 105103 (2008)

11. Martinez-Seara, H., Rog, T., Pasenkiewicz-Gierula, M., Vattulainen, I., Karttunen, M., Reigada, R.: Interplay of unsaturated phospholipids and cholesterol in membranes: Effect of the double-bond position. Biophys. J. 95, 3295-3305 (2008)

12. Grimm, R.L., Beauchamp, J.L.: Dynamics of field-induced droplet ionization: Time-resolved studies of distortion, jetting, and progeny formation from charged and neutral methanol droplets exposed to strong electric fields. J. Phys. Chem. B 109, 8244-8250 (2005)

13. Grimm, R.L., Hodyss, R., Beauchamp, J.L.: Probing interfacial chemistry of single droplets with field-induced droplet ionization mass spectrometry: Physical adsorption of polycyclic aromatic hydrocarbons and ozonolysis of oleic acid and related compounds. Anal. Chem. 78, 3800-3806 (2006)

14. Kim, H.I., Kim, H.J., Shin, Y.S., Beegle, L.W., Jang, S.S., Neidholdt, E.L., Goddard, W.A., Heath, J.R., Kanik, I., Beauchamp, J.L.: Interfacial reactions of ozone with surfactant protein B in a model lung surfactant system. J. Am. Chem. Soc. 132, 2254-2263 (2010)

15. Kim, H.I., Kim, H., Shin, Y.S., Beegle, L.W., Goddard, W.A., Heath, J. R., Kanik, I., Beauchamp, J.L.: Time resolved studies of interfacial reactions of ozone with pulmonary phospholipid surfactants using field induced droplet ionization mass spectrometry. J. Phys. Chem. B 114, 9496-9503 (2010)
16. Schofield, M., Jenski, L.J., Dumaual, A.C., Stillwell, W.: Cholesterol versus cholesterol sulfate: Effects on properties of phospholipid bilayers containing docosahexaenoic acid. Chem. Phys. Lipids 95, 23-36 (1998)

17. Duff, R.B.: 344. Carbohydrate sulphuric esters. Part V. The demonstration of walden inversion on hydrolysis of barium 1: 6-Anhydro- $\beta$-DGalactose 2-Sulphate. J. Chem. Soc. 1597-1600 (1949)

18. Sandhoff, R., Brügger, B., Jeckel, D., Lehmann, W.D., Wieland, F.T.: Determination of cholesterol at the low picomole level by nano-electrospray ionization tandem mass spectrometry. J. Lipid Res. 40, 126-132 (1999)

19. Feller, S.E., MacKerell, A.D.: An improved empirical potential energy function for molecular simulations of phospholipids. J. Phys. Chem. B 104, 7510-7515 (2000)

20. Schlenkrich, M., Brickmann, J., MacKerell Jr., A.D., Karplus, M.: An empirical potential energy function for phospholipids: Criteria for parameter optimization and applications. In: Merz Jr., K.M., Roux, B. (eds.) Biological Membranes: A Molecular Perspective from Computation and Experiment, pp. 31-81. Birkhauser, Boston (1996)

21. Brooks, B.R., Brooks III, C.L., Mackerell Jr., A.D., Nilsson, L., Petrella, R.J., Roux, B., Won, Y., Archontis, G., Bartels, C., Boresch, S., Caflisch, A., Caves, L., Cui, Q., Dinner, A.R., Feig, M., Fischer, S., Gao, J., Hodoscek, M., Im, W., Kuczera, K., Lazaridis, T., Ma, J., Ovchinnikov, V., Paci, E., Pastor, R.W., Post, C.B., Pu, J.Z., Schaefer, M., Tidor, B., Venable, R.M., Woodcock, H.L., Wu, X., Yang, W., York, D.M., Karplus, M.: Charmm: The biomolecular simulation program. J. Comput. Chem. 30, 1545-1614 (2009)

22. Pitman, M.C., Suits, F., MacKerell, A.D., Feller, S.E.: Molecular-level organization of saturated and polyunsaturated fatty acids in a phosphatidylcholine bilayer containing cholesteral. Biochemistry 43, 1531815328 (2004)

23. MacKerell, A.D., Bashford, D., Bellott, M., Dunbrack, R.L., Evanseck, J.D., Field, M.J., Fischer, S., Gao, J., Guo, H., Ha, S., JosephMcCarthy, D., Kuchnir, L., Kuczera, K., Lau, F.T.K., Mattos, C., Michnick, S., Ngo, T., Nguyen, D.T., Prodhom, B., Reiher, W.E., Roux, B., Schlenkrich, M., Smith, J.C., Stote, R., Straub, J., Watanabe, M., Wiorkiewicz-Kuczera, J., Yin, D., Karplus, M.: All-atom empirical potential for molecular modeling and dynamics studies of proteins. $J$. Phys. Chem. B 102, 3586-3616 (1998)

24. Foloppe, N., MacKerell, A.D.: All-atom empirical force field for nucleic acids: I. Parameter optimization based on small molecule and condensed phase macromolecular target data. J. Comput. Chem. 21, 86-104 (2000)

25. Gumulka, J., Smith, L.L.: Ozonization of cholesterol. J. Am. Chem. Soc. 105, 1972-1979 (1983)

26. Dreyfus, M.A., Tolocka, M.P., Dodds, S.M., Dykins, J., Johnston, M. V.: Cholesterol ozonolysis: Kinetics, mechanism, and oligomer products. J. Phys. Chem. A 109, 6242-6248 (2005)

27. Sathishkumar, K., Haque, M., Perumal, T.E., Francis, J., Uppu, R.M.: A major ozonation product of cholesterol, $3 \beta$-Hydroxy-5-Oxo-5,6-Secocholestan-6-Al, induces apoptosis in H9c2 cardiomyoblasts. FEBS Lett. 579, 6444-6450 (2005)

28. Wang, K., Bermúdez, E., Pryor, W.A.: The ozonation of cholesterol: Separation and identification of 2,4-dinitrophenulhydrazine derivatization products of 3ß-Hydroxy-5-Oxo-5,6-Secocholestan-6-Al. Steroids 58, 225-229 (1993)

29. Tagiri-Endo, M., Nakagawa, K., Sugawara, T., Ono, K., Miyazawa, T.: Ozonation of cholesterol in the presence of ethanol: Identification of a cytotoxic ethoxyhydroperoxide molecule. Lipids 39, 259-264 (2004)

30. Martinez, R.I., Herron, J.T., Huie, R.E.: The mechanism of ozonealkene reactions in the gas phase. A mass spectrometric study of the reactions of eight linear and branched-chain alkenes. J. Am. Chem. Soc. 103, 3807-3820 (1981)

31. Pulfer, M., Harrison, K., Murphy, R.: Direct electrospray tandem mass spectrometry of the unstable hydroperoxy bishemiacetal product derived from cholesterol ozonolysis. J. Am. Soc. Mass Spectrom. 15, 194-202 (2004)

32. Karagulian, F., Lea, A.S., Dilbeck, C.W., Finlayson-Pitts, B.J.: A new mechanism for ozonolysis of unsaturated organics on solids: Phosphocholines on Nacl as a model for sea salt particles. Phys. Chem., Chem. Phys. 10, 528-541 (2008)

33. Santrock, J., Gorski, R.A., Ogara, J.F.: Products and mechanism of the reaction of ozone with phospholipids in unilamellar phospholipidvesicles. Chem. Res. Toxicol. 5, 134-141 (1992)

34. Lafont, S., Rapaport, H., Somjen, G.J., Renault, A., Howes, P.B., Kjaer, K., Als-Nielsen, J., Leiserowitz, L., Lahav, M.: Monitoring the nucleation of crystalline films of cholesterol on water and in the presence of phospholipid. J. Phys. Chem. B 102, 761-765 (1998) 
35. Rapaport, H., Kuzmenko, I., Lafont, S., Kjaer, K., Howes, P.B., AlsNielsen, J., Lahav, M., Leiserowitz, L.: Cholesterol monohydrate nucleation in ultrathin films on water. Biophys. J. 81, 2729-2736 (2001)

36. Pulfer, M.K., Murphy, R.C.: Formation of biologically active oxysterols during ozonolysis of cholesterol present in lung surfactant. J. Biol. Chem. 279, 26331-26338 (2004)

37. von Gunten, U.: Ozonation of drinking water: Part I. Oxidation kinetics and product formation. Water Res. 37, 1443-1467 (2003)

38. Pryor, W.A.: Mechanisms of radical formation from reactions of ozone with target molecules in the lung. Free Radic. Biol. Med. 17, 451-465 (1994)

39. Seinfeld, J.H., Pandis, S.N.: Atmospheric Chemistry and Physics: From Air Pollution to Climate Change. John Wiley and Sons, Inc., New York (1998)
40. Nakahara, H., Nakamura, S., Nakamura, K., Inagaki, M., Aso, M., Higuchi, R., Shibata, O.: Cerebroside langmuir monolayers originated from the echinoderms: Ii. Binary systems of cerebrosides and steroids. Colloid Surf. B-Biointerfaces 42, 175-185 (2005)

41. Kaznessis, Y.N., Kim, S., Larson, R.G.: Specific mode of interaction between components of model pulmonary surfactants using computer simulations. J. Mol. Biol. 322, 569-582 (2002)

42. Baoukina, S., Monticelli, L., Risselada, H.J., Marrink, S.J., Tieleman, D.P.: The molecular mechanism of lipid monolayer collapse. Proc. Natl. Acad. Sci. U.S.A. 105, 10803-10808 (2008)

43. Kaznessis, Y.N., Kim, S.T., Larson, R.G.: Simulations of zwitterionic and anionic phospholipid monolayers. Biophys. J. 82, 1731-1742 (2002) 